\title{
HÁBITOS SEXUAIS E PRÁTICA CONTRACEPTIVA DOS ACADÊMICOS DE UMA UNIVERSIDADE PÚBLICA NO NORTE DE MATO GROSSO
}

\section{SEXUAL HABITS AND CONTRACEPTIVE PRACTICE OF ACADEMICS FROM A PUBLIC UNIVERSITY IN NORTHERN MATO GROSSO}

\author{
Cezar Augusto da Silva Flores', Andréia Dulciane Petter², \\ Suellen Rodrigues de Oliveira Maier', Patrícia Reis Souza ${ }^{3}$
}

\section{RESUMO}

Objetivo: O presente estudo teve como objetivo elucidar os hábitos sexuais dos acadêmicos matriculados na Universidade Federal de Mato Grosso, campus Universitário de Sinop e o uso de contraceptivos e preservativos entre os estudantes. Tratou-se de uma pesquisa descritiva de abordagem quantitativa, aprovado pelo Comitê de Ética em Pesquisa do Hospital Universitário Júlio Muller, parecer número 1.072.649/2015. A coleta de dados ocorreu através de questionário autoaplicável contendo 10 questões. Foram incluídos no estudo 135 acadêmicos, matriculados no período letivo de 2015/2. Grande parte dos entrevistados fica exposta a situações de risco de contrair uma IST, uma vez que o uso do método contraceptivo não ocorre em todas as relações sexuais. Os resultados obtidos reforçam a necessidade de investimentos na educação da população e dos acadêmicos em geral, no que se refere às informações necessárias para a utilização correta dos métodos contraceptivos.

Descritores: Inquéritos sobre o Uso de Métodos Contraceptivos; Doenças Sexualmente Transmissíveis; Educação em Enfermagem.

\begin{abstract}
Objective: This study aimed to elucidate the sexual habits of students enrolled at the Federal University of Mato Grosso, Sinop University campus and the use of contraceptives and condoms among students. This was a descriptive quantitative approach approved by the Ethics Committee of the University Hospital Júlio Muller, opinion number 1072649/2015. The data were collected through self-administered questionnaire containing 10 questions. The study included 135 students enrolled in the school year of 2015/2. Most respondents are exposed to situations of risk of contracting an STI, since the use of contraception does not occur in all sexual relations. The results reinforce the need for investments in public education and academics in general, with regard to the necessary information for the correct use of contraceptive methods.
\end{abstract}

Descriptors: Contraceptive Prevalence Surveys; Sexually Transmitted Diseases; Education, Nursing.
${ }^{1}$ Mestre em Educação pela Universidade Federal de Mato Grosso (UFMT), Cuiabá, MT, Brasil.

${ }^{2}$ Graduada em Enfermagem pela Universidade Federal de Mato Grosso (UFMT), Sinop, MT, Brasil.

${ }^{3}$ Doutora em Ciências pela Universidade de São Paulo (USP), Ribeirão Preto, SP, Brasil. 


\section{Introdução}

O acesso às informações no que concerne à sexualidade e à prática sexual tem ocorrido, precocemente, por parte de adolescentes, que movidos pela curiosidade e/ou busca de prazer acabam tendo a primeira relação sexual. Tal fato pode promover um aumento nos índices de Infecções Sexualmente Transmissíveis (IST) e de gestações não planejadas entre os jovens, em especial os que ainda encontram-se em idade escolar ${ }^{1}$.

Considerando o elevado número de jovens com Síndrome da Imunodeficiência Adquirida (AIDS), é de suma importância que seja estimulado o uso de práticas contraceptivas com dupla proteção, para que haja prevenção tanto de gestação não planejada quanto de infecções pelo Vírus da Imunodeficiência Humana/ Síndrome da Imunodeficiência Adquirida (HIVIAIDS) e por outras ISTs².

A prevenção pode ser realizada com o uso dos preservativos (masculino ou feminino), ou ainda, há alternativa de utilizá-los em associação com outros métodos contraceptivos como, anticoncepcional, dispositivo intra-uterino (DIU), tabelinha ou pílula do dia seguinte, ficando tal escolha a critério de cada indivíduo ou de ambos os parceiros².

Diante do exposto, observa-se a necessidade da conscientização dos jovens sobre a prática da relação sexual segura, incentivando o uso do preservativo e outros métodos contraceptivos em todas as relações sexuais.

Vale ressaltar que os métodos anticoncepcionais são distribuídos gratuitamente em todas as Unidades Básicas de Saúde (UBS), uma informação de grande relevância, uma vez que parte da população não possui condições financeiras para adquirir tais métodos, ou ainda, desconhece o fornecimento pela rede básica de saúde².

A grande preocupação em relação às ISTs está no seu poder de disseminação e os danos que as mesmas podem provocar na saúde da população como, distúrbios emocionais graves, doença inflamatórias pélvicas, lesões fetais, infertilidade, câncer, proliferação do vírus HIV e até mesmo a morte ${ }^{4}$. A transmissão ocorre quando uma pessoa contaminada com determinado tipo de doença, seja ela viral ou bacteriana, tem relação sexual desprotegida com outra pessoa sadia-5.

Com o objetivo de prevenir a gravidez não planejada, são disponibilizados no mercado diversos métodos contraceptivos, os quais podem ser classificados em métodos de barreira, substâncias medicamentosas ou procedimentos cirúrgicos. Tais métodos podem ser utilizados para a prevenção temporária ou definitiva da gestação. Além disso, é essencial que antes de fazer uso de qualquer método contraceptivo o indivíduo ou o casal conheçam todos os métodos disponíveis para posteriormente escolherem o método que melhor se adaptam.

É fundamental que jovens e a população geral obtenham as informações corretas e tenham acesso a diferentes métodos contraceptivos, entretanto, além disso, é essencial que façam o uso correto dos mesmos. Caso a assistência à saúde não seja realizada corretamente, o conhecimento da população referente aos métodos se torna inadequado o que predispõe a erros no uso, e também, culminam com a resistência dos usuários ao programa de prevenção as doenças ${ }^{6}$.

Sabe-se que quanto maior o nível de escolaridade, mais tarde se dá o início da atividade sexual, o que demonstra que o elevado nível de instrução torna-se um fator para que a primeira relação sexual seja adiada, o que também influencia no uso imediato de métodos contraceptivos desde o início de tais relações. Deste modo, conclui-se que a ausência da utilização de algum método contraceptivo está associada à falta de informação.

Sob este prisma, vislumbrou-se elucidar os hábitos sexuais dos acadêmicos matriculados na Universidade Federal de Mato Grosso, campus Universitário de Sinop e o uso de contraceptivos e preservativos entre os estudantes.

\section{Metodologia}

Trata-se de um estudo epidemiológico, descritivo, com abordagem quantitativa, com vistas a responder ao objetivo proposto anteriormente. Esta pesquisa foi aprovada pelo Comitê de Ética e Pesquisa do Hospital Júlio Muller da Universidade Federal do Mato Grosso, sob o protocolo de $n^{\circ} 1.072 .649$ em 10/06/2015.

Para a coleta de dados foi elaborado um questionário autoaplicável com questões abertas e fechadas, que abordavam sobre a idade, gênero sexual, estado civil, atividade sexual, conhecimento sobre métodos contraceptivos, métodos contraceptivos utilizados e o motivo da utilização, ou não, de tais métodos. Esta etapa aconteceu no mês de dezembro de 2015, em seguida os dados foram tabelados, quantificados e analisados utilizando-se de estatística simples.

A escolha em realizar a pesquisa no quinto semestre de cada curso, se deu pela intenção de abordar os acadêmicos que estavam cursando a metade de cada curso, uma vez que os cursos que participaram da pesquisa têm um período de integralização mínimo de 9 a 10 semestres, e o total de 220 acadêmicos matriculados. 
Participaram do estudo 135 acadêmicos, devidamente matriculados no $5^{\circ}$ período dos cursos de Agronomia, Farmácia, Enfermagem, Licenciatura em Ciências Naturais, Medicina Veterinária, Engenharia Agrícola e Ambiental, Engenharia Florestal e Zootecnia da Universidade Federal do Mato Grosso, campus Universitário de Sinop, no período letivo de 2015/2, sendo que 85 discentes não concordaram em participar do estudo ou não estavam frequentando regularmente as disciplinas do referido semestre.

A coleta de dados ocorreu em sala de aula e após assinatura do Termo de Consentimento Livre e Esclarecido (TCLE), foram entregues os questionários autoaplicáveis, somente aos acadêmicos que estavam em sala de aula no momento da abordagem. Após serem respondidos, os questionários foram devolvidos aos pesquisadores.

A análise de dados foi realizada a partir de estatística simples, com a agregação dos resultados obtidos, de modo a refletir o hábito sexual e as práticas contraceptivas no local do estudo. Para tal, os dados foram tabulados e lançados um uma planilha do programa Microsoft Excel para melhor armazenamento e apresentados em forma de gráficos e de tabelas, a fim de trazer a realidade local.

\section{Resultados e Discussão}

Em relação ao sexo predominou o gênero feminino com um total de 74 acadêmicas contra 61 acadêmicos do sexo masculino. A variável predominante em relação à idade foi entre 18 e 24 anos, seguido de 25 a 30 anos, acompanhado de 31 a 37 anos, e por fim de 38 a 45 anos, como demonstrado na tabela 1.

Tabela 1- Distribuição dos acadêmicos por faixa etária

\begin{tabular}{c|c|c}
\hline Idade & $\mathbf{N}^{\circ}$ estudantes & $\%$ \\
\hline 18 a 24 anos & 101 & 75 \\
\hline 25 a 30 anos & 26 & 19 \\
\hline 31 a 37 anos & 5 & 4 \\
\hline 38 a 45 anos & 3 & 2 \\
\hline Total & $\mathbf{1 3 5}$ & $\mathbf{1 0 0 \%}$ \\
\hline
\end{tabular}

Fonte: Banco de dados dos autores (2016).

A maioria dos acadêmicos são considerados jovens, tal fato caracteriza um dado importante, uma vez que os mesmos estão saindo da adolescência e entrando na vida adulta, vivenciando novas experiências e muitas vezes sem as informações necessárias para sua proteção.

Muitos adolescentes iniciam sua vida sexual próximo ao ingresso na vida universitária, esses dois eventos representam um marco em direção à autonomia e à independência própria da vida adulta ${ }^{8}$.

Quando questionados sobre o seu estado civil, observamos a distribuição dos acadêmicos conforme demonstrado na Tabela 2.

Tabela 2 - Distribuição dos acadêmicos por estado civil

\begin{tabular}{c|c|c}
\hline Estado Civil & $\mathbf{N}^{\circ}$ estudantes & $\%$ \\
\hline Solteiro & 112 & 83 \\
\hline Casado & 17 & 13 \\
\hline Convivente & 6 & 4 \\
\hline Total & $\mathbf{1 3 5}$ & $\mathbf{1 0 0 \%}$ \\
\hline
\end{tabular}

Fonte: Banco de dados dos autores (2016). 
Vale ressaltar que dos $83 \%$ que se declaram solteiros, 34\% estão namorando atualmente, portanto $49 \%$ dos acadêmicos não tem parceiro(a) sexual fixo, sendo que esses estão mais propensos à infecção por IST/AIDS do que os que estão namorando ou são casados/convivente, uma vez que os solteiros possuem uma maior probabilidade de exposição a relações sexuais com outros ou múltiplos parceiros ${ }^{8}$.

Em relação à atividade sexual, 95,55\% acadêmicos já iniciaram atividade sexual e 4,45\% acadêmicos nunca tiveram relação sexual, dados que permitem afirmar que a maioria dos universitários são sexualmente ativos.

Uma das questões listava dez alternativas, a saber: preservativo (camisinha), coito interrompido, tabelinha, temperatura basal, vasectomia, pílula anticoncepcional, muco cervical, DIU, diafragma e laqueadura, a qual tinha como finalidade avaliar o conhecimento dos acadêmicos sobre os métodos contraceptivos, em que os mesmos deveriam assinalar todas as alternativas que consideravam como um método contraceptivo.

De acordo com a Figura 1, os métodos contraceptivos mais conhecidos foram: Pílula anticoncepcional com 121 acadêmicos citando tal método (89\%), e 118 acadêmicos que citaram o preservativo (87\%). Pode-se perceber que 0 preservativo é um dos contraceptivos de escolha entre os acadêmicos participantes da pesquisa, embora a preferência por este esteja ligada a outros fatores, que muitas vezes não é a proteção contra as IST/HIVIAIDS. Observou-se que a proporção de usuários de pílula anticoncepcional aumenta entre os participantes que responderam estar namorando, indicando a troca do uso do preservativo por pílula anticoncepcional, destacando que tal fato ocorre quando há o estabelecimento de uma confiança pela relação fixa e amorosa compromissada, onde o uso do preservativo tende a ser interrompido ${ }^{10}$.

Por outro lado, observa-se também o conhecimento de métodos contraceptivos de baixa eficácia, como é o caso da tabelinha sendo citado por 30 acadêmicos (22\%), e do coito interrompido citado por 10 acadêmicos (7\%); métodos que necessitam de amplo conhecimento da fisiologia, para que possam ser utilizados com eficácia ${ }^{11}$.

Quando questionados sobre os métodos contraceptivos reversíveis e os definitivos, apresentaram conhecimento significativo, como apresentado na Figura 1. Entretanto na pergunta de número oito (08) do questionário, nenhum estudante relatou o uso de métodos definitivos, acredita-se que não tenham ocorrido relatos de tais métodos por possuírem idade entre 18 a 24 anos, considerados ainda jovens para realizar um método contraceptivo definitivo.

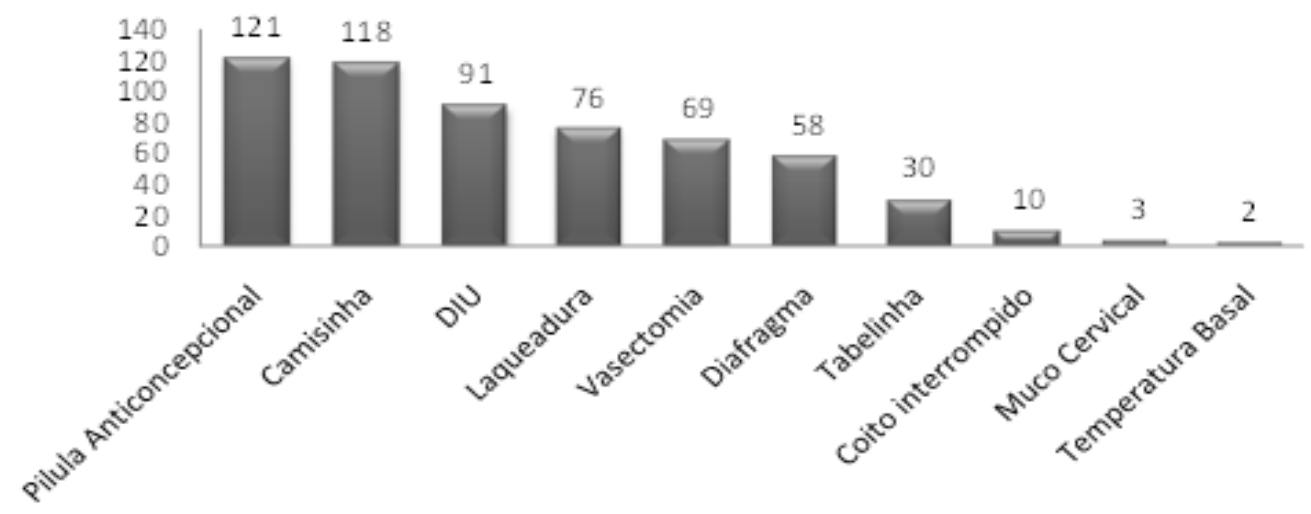

Figura 1 - Distribuição dos acadêmicos sexualmente ativos UFMT/Sinop de acordo com os conhecimentos sobre os métodos contraceptivos.

Os acadêmicos demonstraram pouco conhecimento quanto aos métodos de temperatura basal e do muco cervical, sendo citados por apenas dois e três estudantes, respectivamente (1\% e $2 \%)$, tais dados representam uma estatística que hipoteticamente reflete ao não uso de tais métodos, o que pode ser considerado uma atitude positiva uma vez que os mesmos apenas têm o intuito de prevenir a gestação não planejada, e assim são considerados como métodos não confiáveis, em que a taxa de prevenção contra as IST é nula; esses métodos devem ser evitados por terem altas chances de contaminação por IST ${ }^{9}$.

Esses resultados demonstram que os acadêmicos possuem conhecimento sobre os métodos contraceptivos, e verifica-se que o conhecimento sobre esses métodos está ligado positivamente com os conhecimentos sobre as IST e com as atitudes de prevenção, diminuindo o comportamento de risco ${ }^{12}$. Desta forma, faz-se necessária à implementação de estratégias que permitam a esses jovens graduandos conscientizar-se sobre a importância dessas associação ${ }^{13}$. 
Quando indagados sobre de quem é a responsabilidade em usar os métodos anticoncepcionais, a maioria dos acadêmicos respondeu que é tanto do homem quanto da mulher, representando 121 acadêmicos $(89,6 \%)$. No entanto, sete acadêmicos $(5 \%)$ acreditam que a responsabilidade seja principalmente da mulher, dois (1.4\%) responderam que a responsabilidade é principalmente do homem e $11(8,1 \%)$ informam que depende do momento e da necessidade de utilização de determinados métodos.

Tais resultados mostram que parece haver certo desencontro entre compreender as responsabilidades de homens e mulheres, relacionadas ao controle de reprodução. Reforça ainda a mulher como a principal responsável pelas questões reprodutivas, uma vez que a assistência está voltada, predominantemente, para ela, não oferecendo oportunidades de participação masculina ${ }^{14}$.

A Figura 2 apresenta a frequência do uso de métodos contraceptivos, observa-se que tais dados são preocupantes, já que foi demonstrado que grande parte dos entrevistados fica exposta a situações de risco de contrair uma IST, uma vez que o uso do método contraceptivo não ocorre em todas as relações sexuais. Podemos observar que apesar das mulheres possuírem conhecimento sobre os riscos da exposição sexual desprotegida, elas não usam o preservativo em relacionamento estável devido à ausência de poder de decisão, e por questões relacionadas à fidelidade ${ }^{15}$.

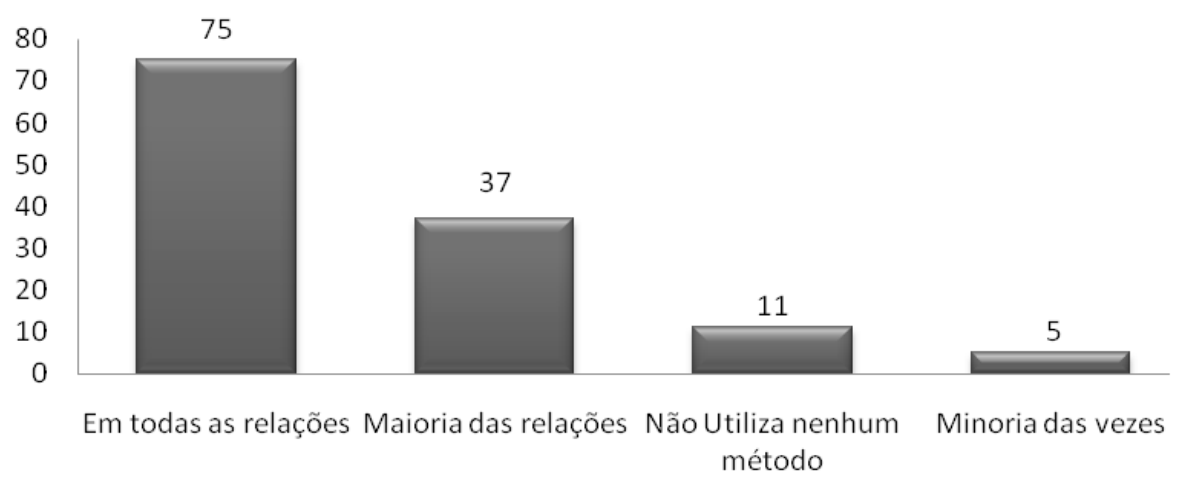

Figura 2 - Frequência do uso de métodos contraceptivos pelos acadêmicos UFMT/Sinop.

Já a Figura 3 demonstra qual o método contraceptivo utilizado, 96 (53\%) dos entrevistados responderam que utilizam preservativo, 35 (59\%) pílula anticoncepcional, 18 (10\%) pílula do dia seguinte, e oito (4\%) responderam que utilizam outros métodos, entre eles o método Billings, anticoncepcional injetável, coito interrompido e a não utilização de métodos contraceptivos por crenças religiosas.

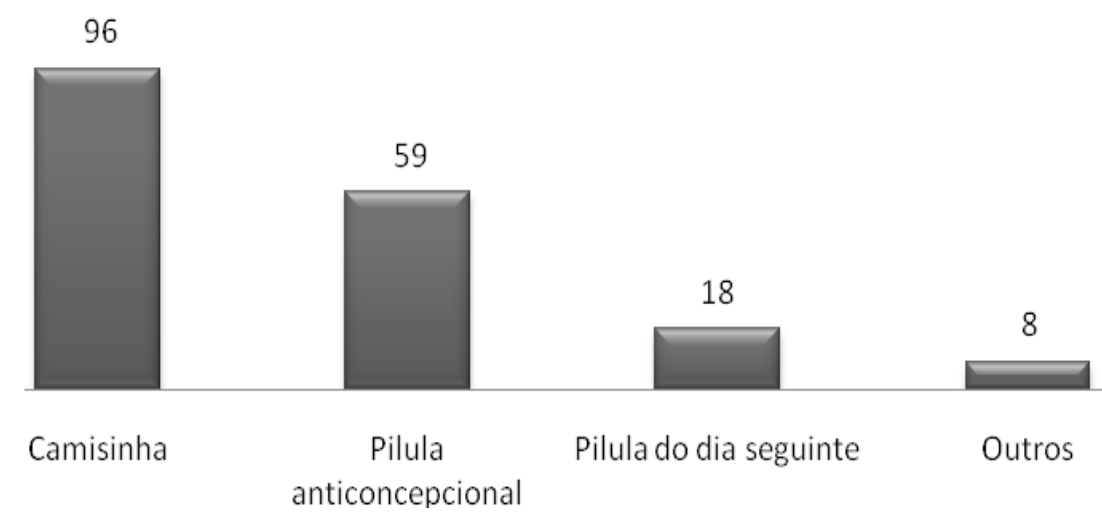

Figura 3 - Descrição do método anticoncepcional utilizado pelos acadêmicos UFMT/Sinop. 
O método Billings se assemelha ao método do muco cervical, que consiste na verificação das características do muco cervical. 0 coito interrompido e a não utilização por motivos religiosos, também foram citados, o que aumenta 0 fator de risco de exposição às ISTs, já que os mesmos podem ser utilizados apenas como prevenção de gestações não planejadas, e não como método de prevenção contra as ISTs ${ }^{16}$.

O coito interrompido é um dos métodos menos eficaz, um método usuário dependente, sendo o risco de gravidez maior no caso de o homem não retirar o pênis da vagina antes da ejaculação, ocorrendo cerca de 27 gestações por 100 mulheres cujo parceiro utiliza o coito interrompido no primeiro ano; portanto, a maioria dos acadêmicos mostrou conhecimento correto sobre o mesmo ${ }^{17}$.

Um participante do estudo relatou não fazer o uso de nenhum método contraceptivo por ser "homofóbica", o que demonstra certo desconhecimento sobre o assunto, tanto sobre homofobia quanto para o uso de métodos contraceptivos, o que limitou a interpretação da resposta.

Perguntamos qual motivo de escolherem o método anticoncepcional utilizado e os resultados demonstraram que os principais motivos citados foram a prevenção de IST/AIDS e a gestação não planejada como pode-se observar na Figura 4.

100

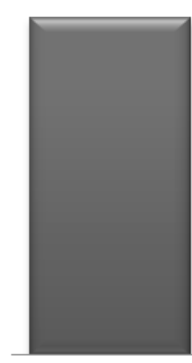

Prevenção IST
99

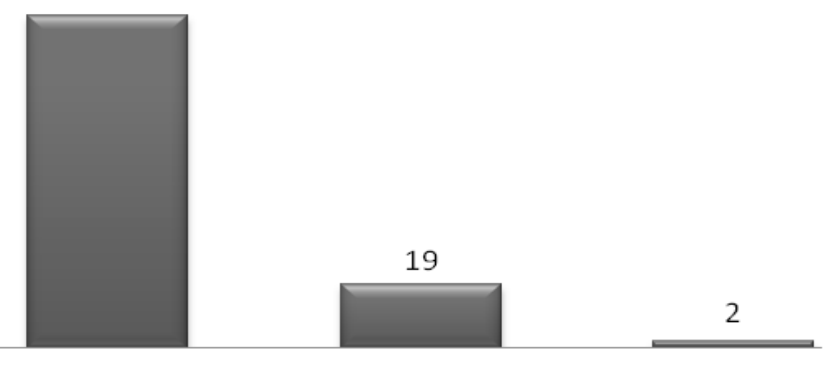

Outros

Figura 4 - Motivo da escolha do método anticoncepcional utilizado pelos acadêmicos UFMT/Sinop entrevistados. *IST: Infecção Sexualmente Transmissível

O preservativo, tanto masculino quanto feminino, é o método mais eficaz para a proteção contra a transmissão de IST/AIDS, além de oferecer proteção adicional como contraceptivo, se constituindo o único método que oferece essa dupla efetividade ${ }^{8}$.

Os dados mostram que embora os acadêmicos apresentem práticas voltadas para a prevenção de IST/AIDS a preocupação maior continua sendo a gravidez não planejada, essas dão lugar a práticas menos seguras, em algumas situações, principalmente quando as atividades sexuais ocorrem em relações estáveis, esquecendo das relações antecedentes dos parceiros e acreditando na fidelidade do mesmo.

Quando perguntado se já haviam entrado em contato com algum tipo de IST, apenas cinco acadêmicos (4\%) responderam que sim, e todos citaram que obtiveram contaminação por herpes genital. Quando comparadas as respostas desses cinco entrevistados que já apresentaram IST, três foram do sexo feminino e dois do sexo masculino. A idade variou entre 19 e 30 anos. Quanto ao estado civil, quatro são solteiros e apenas um é casado. Os cinco concordam que a responsabilidade em usar os métodos contraceptivos é tanto do homem quando da mulher. Quatro afirmaram que usam algum tipo de método contraceptivo na maioria das relações e um respondeu que usa na minoria das vezes. Quanto ao método utilizado, dois responderam que usam preservativos e pílula anticoncepcional, dois responderam que usam somente preservativos e um utiliza do método Billings.

Dado o constrangimento gerado quando se aborda o tema "aquisição das IST" e também o desconhecimento sobre sua ocorrência, obteve-se um reduzido número de pessoas que afirmaram ter adquirido uma IST, o que impossibilitou melhores interpretações. Acreditasse que nesta pesquisa os entrevistados possam ter omitido sobre uma possível contaminação, por ser um dado extremamente pessoal, em que são englobados muitos mitos e preconceitos. 


\section{Considerações Finais}

Podemos concluir que mesmo lidando com pessoas de um maior grau de escolaridade, ainda se faz necessária a implantação de políticas educacionais quando se diz respeito à sexualidade, visando à orientação desses jovens acadêmicos quanto às práticas sexuais seguras, a fim de reduzir a incidência de IST/AIDS e gravidez não planejada e tornar essa população mais responsável, atenta e seletiva quanto aos cuidados com a sua saúde e a dos seus parceiros. Além disso, a educação em saúde sexual pode promover a disseminação de informações confiáveis e consequentemente diminuir a exposição dos jovens a riscos que prejudiquem a saúde.

Sabemos que somente a informação não é suficiente, é necessário conhecer os hábitos e o que essa população realmente pensa e deseja, para constatar se existem lacunas nas ações de saúde e educação voltadas para a vida sexual e reprodutiva dos jovens, chamando a atenção para a importância de que se aposte na educação sexual como estratégia da saúde sexual e reprodutiva.

Os resultados obtidos reforçam a necessidade de investimentos na educação dos acadêmicos e da população geral. Principalmente no que se refere à formação do cidadão, capacitando-o para lutar pelos seus direitos, entre os quais o acesso a informações necessárias para a prática da utilização de métodos contraceptivos, sejam eles para prevenção de IST/HIV ou da gestação não planejada.

\section{Referências}

1. BRASIL. Ministério da Saúde, Secretaria de Atenção à Saúde, Departamento de Ações Programáticas Estratégicas. Direitos sexuais, direitos reprodutivos e métodos anticoncepcionais. Brasília2009; 2: 1-52. Disponível em: <http://bvsms. saude.gov.br/bvs/publicacoes/direitos_sexuais_reprodutivos_metodos_anticoncepcionais.pdf>. Acesso 28 mar de 2015. 2. BRASIL, Ministério da Saúde, Secretaria de Políticas de Saúde, Área Técnica de Saúde da Mulher. Assistência em Planejamento Familiar: Manual Técnico. Brasília 2002; 4(40): 1-150. Disponível em: <http://bvsms.saude.gov.br/bvs/ publicacoes/0102assistencia1.pdf>. Acesso em 05 abr 2015.

3. SPINDOLAT, PIMENTEL MRRA, BARROS AS, FRANCO VQ, FERREIRALEM. Produção de conhecimento acerca das doenças sexualmente transmissíveis na população jovem: pesquisa bibliométrica. J. res.: fundam. care. online [periódico na Internet]. 2015 jul/set [citado 2016 maio 10]; 7(3): 3037-3049. Disponível em: http://www.seer.unirio.br/index.php/ cuidadofundamental/article/view/4644/pdf_1668. doi: 10.9789/2175-5361.2015.v7i3.3037-3049.

4. VIANA LC, GEBER S. Ginecologia.3 edição. Editora MedBooc, Rio de Janeiro, 2012.

5. BRASIL. Ministério da Saúde, Secretaria de Atenção à Saúde, Departamento de Atenção Básica. Saúde sexual e saúde reprodutiva.- Brasília 2009; 22: 1-300. Disponível em: <http://www.pim.saude.rs.gov.br/a_PIM/noticias/542/CAB_ Saude_Sextual_e_Reprodutiva.pdf>. Acesso em 31 mar 2015.

6. LOPEZ LM, GREY TW, TOLLEY EE, CHEN M. Brief educational strategies for improving contraception use in young people. Cochrane Library. [periódico na Internet]. 2016 Mar [citado 2016 jun 20]. Disponível em: http://onlinelibrary.wiley. com/doi/10.1002/14651858.CD012025.pub2/abstract;jsessionid=0BDF3AE0404AB835881572A552C14B49.f01t02. doi: 10.1002/14651858.CD012025.pub2.

7. COSTA GPO, GUERRA AQS, ARAÚJO ACPF. Conhecimentos, atitudes e práticas sobre contracepção para adolescentes. J. res.: fundam. care. [período na Internet]. 2016 jan/mar [citado 2016 Fev 01]; 8(1): 3597-3608. Disponível em: http://seer.unirio.br/index.php/cuidadofundamental/article/view/3131/pdf_1763. doi: 10.9789/2175-5361.2016. v8i1.3597-3608.

8. SOUZA CP, PIANTINO CB, QUEIROZ CA, MAIA MAC, FORTUNA CM, ANDRADE RD. Incidência de transmissão vertical do HIV entre gestantes soropositivas cadastradas em um serviço de referência regional. J. res.: fundam. care. [período na Internet]. 2016 abr/jun [citado 2016 abr 07]; 8(2): 4526-4537. Disponível em: http://www.seer.unirio.br/index. php/cuidadofundamental/article/view/4964/pdf_1911. doi: 10.9789/2175-5361.2016.v8i2.4526-4537.

9. SILVACD, MARTINS GDM, FONSECAAD, GOMES VLO. Métodos Contraceptivos: conhecimento e prática de formandos em enfermagem.Revenferm UFPE [periódico na Internet]. 2013 nov [citado 2016 fev 23]; 7(11):6322-8. Disponível em http://www.revista.ufpe.br/revistaenfermagem/index.php/revista/article/download/5048/7666. doi.: 10.5205/reuol.379432322-1-ED.0711201302. 
10. MORRIS JL, RUSHWAN H. Adolescent sexual and reproductive health: The global challenges. International Journal of Gynecology and Obstetrics. [periódico na Internet]. 2015 fev [citado 2016 Jul 01]; 131: S40-S42. Disponível em: http:// www.ijgo.org/article/S0020-7292(15)00085-5/pdf. doi: http://dx.doi.org/10.1016/j.ijgo.2015.02.006.

11. DELATORRE MZ, DIAS ACG. Conhecimento e prática sobre métodos contraceptivos em estudantes universitários. Revista da SPAGESP. [periódico na Internet]. 2015 [citado 2016 jan 28]; 16(1): 60-73. Disponível em: http://pepsic. bvsalud.org/pdf/rspagesp/v16n1/v16n1a06.pdf.

12. LUNA IT, COSTA AGM, COSTA MS, ALVES MDS, VIEIRA NFC, PINHEIRO PNC. Conhecimento e Prevenção das doenças sexualmente transmissíveis entre os adolescentes em situação de rua. Cienc Cuid Saúde. [período na Internet]. 2013 abr/jun [citado 2016 jul 10]; 12(2): 346-355. Disponível em: http://www.revenf.bvs.br/pdf/ccs/v12n2/19.pdf. doi: 10.4025/cienccuidsaude.v12i2.18693.

13. DANTAS KTB, SPÍNDOLAT, TEIXEIRA SVB, LEMOS ACM, FERREIRA LEM. Jovens universitários e o conhecimento acerca das doenças sexualmente transmissíveis - contribuição para cuidar em enfermagem. J. res.: fundam. care. [periódico na Internet]. 2015 jul/set [citado 2016 mar 01]; 7(3): 3020-3026. Disponível: http://www.seer.unirio.br/index. php/cuidadofundamental/article/view/4689/pdf_1666. doi: 10.9789/2175-5361.2015.v7i3.3020-3036.

14. MORAIS ACB, FERREIRAAG, ALMEIDA KL, QUIRINO GS. Participação masculina no planejamento familiar e seus fatores intervenientes. Rev Enfermagem UFSM. [periódico na Internet]. 2014 jul/set [citado 2016 jun 25]; 4(3): 498-508. Disponível em: http://periodicos.ufsm.br/index.php/reufsm/article/view/9998/pdf. doi.: 10.5902/217976929998.

15. OLIVEIRA JG, ARAÚJO JL, ALCHIERI JC, PEREIRA AKAM, NASCIMENTO EGC, VASCONCELOS RB. Conhecimento e comportamento sexual dos universitários diante a vulnerabilidade ao HIVIAIDS. Revista Baiana da Saúde Pública. [periódico na Internet]. 2013 jul/set [citado 2016 mar 23]; 37(3): 702-724. Disponível em: http://files.bvs. br/upload/S/0100-0233/2013/v37n3/a4470.pdf.

16. UCHIMURA NS, UCHIMURATT, ALMEIDA LMM, PEREGO DM, UCHIMURA LYT. Conhecimento, aceitabilidade e uso do método billings de planejamento familiar natural. Rev Gaúcha Enferm. [periódico na Internet] 2011 set [citado 2016 fev 07]; 32(3): 516-23. Disponível em http://www.scielo.br/scielo.php?script=sci_arttext\&pid=S1983-14472011000300012. doi: dx.doi.org/10.1590/S1983-14472011000300012.

17. Organização Mundial de Saúde; Escola Bloomberg de Saúde Pública; Centro de Programas de Comunicação da Universidade Johns Hopkins. Planejamento Familiar: Um Manual Global para Prestadores de Serviços de Saúde. Baltimore e Genebra: CPC e OMS, 2007. Disponível em <http://www.reprolatina.org.br/site/pdfs/HANDBOOK_PORT. pdf> acesso 23 fev 2016.

\section{Cezar Augusto da Silva Flores}

Endereço para correspondência - Rua: Sicília, $n^{\circ}: 794$, Bairro: Residencial Florença, CEP: 78555-402, Sinop, MG, Brasil.

E-mail: cezarflores2010@gmail.com Lattes: http://lattes.cnpq.br/3840945833187621

Andréia Dulciane Petter - andreia_petter@hotmail.com Suellen Rodrigues de Oliveira Maier - suellen_enf2004@hotmail.com Patrícia Reis Souza - patriciareisenfermagem@hotmail.com

\section{Enviado em 30 de maio de 2016. Aceito em 23 de setembro de 2016.}

